\title{
Percent of longitudinal body contraction and latency of response onset in planaria as a function of intensity of electric shock
}

\author{
H. D. KIMMEL, S. J. OLSON, J. M. STEPHENSON, and R. L. RAY \\ University of South Florida, Tampa, Florida 33620
}

\begin{abstract}
A videotape technique was used to obtain two quantitative measures of longitudinal body contraction of four planaria (Dugesia dorotocephala) elicited by five shock intensities, delivered four times each over 5 successive days. Percent of longitudinal contraction and response onset latency both increased significantly as a function of shock intensity. Contractions increased with increases in intensity and then tended to reduce at the highest intensity. Latency reduced monotonically with intensity increase. It was concluded that both measures could be used productively in habituation and conditioning studies with planaria.
\end{abstract}

The use of videotape recording of behavioral reactions of planaria (Dugesia dorotocephala) to obtain quantitative measures of response strength has recently been described by Kimmel, Ray, and King (1975). These authors have shown that reliable and relatively consistent measures may be obtained of percent of longitudinal body contraction, from stimulus onset to point of maximum contraction, and of response latency, from onset of stimulus to onset of response. It was suggested that the use of quantitative response measures of this type would facilitate efforts to relate basic behavioral phenomena in other preparations with those seen in planaria.

The present study sought to determine whether either percent of longitudinal contraction or response latency was related systematically to the intensity of an electric shock stimulus used to elicit responses. The assumption was made that such a relationship would have to be demonstrated empirically before either measure could be considered useful for basic conditioning and/or habituation research.

The basic equipment and measurement techniques have been presented in detail by Kimmel, Ray, and King (1975). Briefly, the worm swims in spring water in a watch crystal which is mounted directly above a TV camera. Four stainless steel shock electrodes, adjacent polarities opposite, are placed $90^{\circ}$ apart around the circumference of the swimming space, delivering the $200-\mathrm{Hz}$ output of an audio oscillator via an $\mathrm{R}-\mathrm{C}$ phase shift network which generates a relatively homogeneous electrical field throughout the water. Stimulus duration was $0.2 \mathrm{sec}$, with five different intensities, each delivered four times each day for 5 consecutive days. Within each day, orders of intensities were randomized, with the restriction that no single intensity occur twice before each of the others accurred once. The intensities used were: $3.7,4.4,5.0,5.8$, and $6.2 \mathrm{~V}$ ac. ${ }^{1}$ Stop action and manual movement of the videotape were employed to measure longitudinal body length at shock onset and point of maximum contraction (these are then converted to percent of longitudinal contraction) and to count the number of synchronizing pulses on the videotape (separated by $0.0167 \mathrm{sec}$ ) between stimulus and response onsets, producing response latency measures. The data shown in Figures 1 and 2 are averaged from four planaria run as described above. $^{2}$

Figure 1 presents the average percent of longitudinal contraction as a function of shock intensity. As can be seen in this figure, percent of contraction first increased as shock intensity increased and then declined somewhat at the highest intensity used. Analysis of variance of these data indicated that the overall effect of intensity was statistically significant, $F(4,12)=4.84, p<.05$.

Figure 2 shows the average latency of the response to shock as a function of shock intensity. Here, a

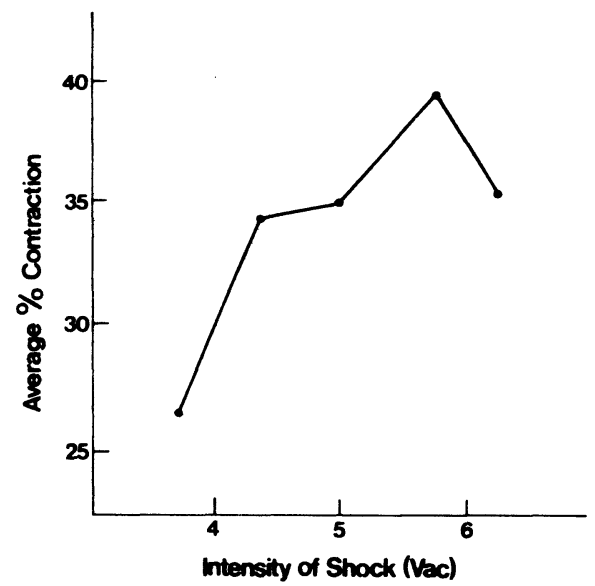

Figure 1. Average percent longitudinal body contraction elicited by shock as a function of shock intensity. See text for actual intensities employed. 


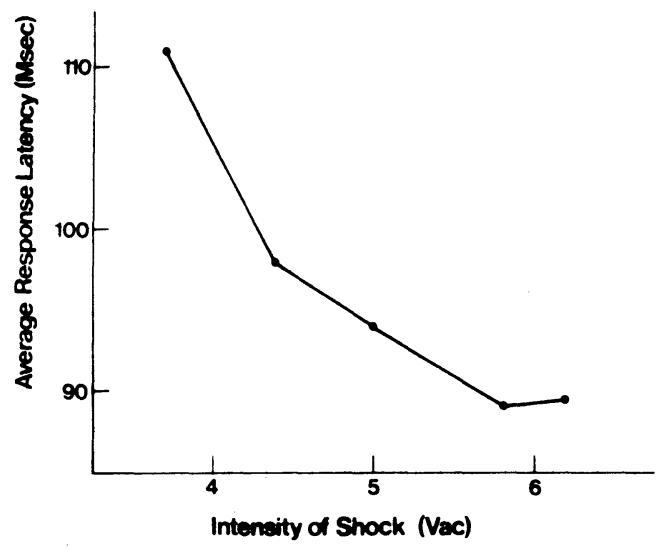

Figure 2. Average response onset latency of shock-elicited responses as a function of shock intensity. See text for actual intensities employed.

monotonic decline in latency was seen as intensity increased. Analysis of variance of the latency measures showed that the effect of shock intensity was again significant, $F(4,12)=5.98, p<.05$.

Both of the quantitative measures described above are reliably influenced in a systematic fashion by manipulation of shock intensity. The monotonic negative relationship between shock intensity and response onset latency, shown in Figure 2, was similar in form to intensity-latency functions obtained for single cortical neurons by Hind, Goldberg, Greenwood, and Rose (1963) as well as for peripheral human autonomic responses by Uno and Grings (1965). It is of some interest to note also that the range of onset latencies of body contractions to shock in our planaria was quite similar to that of the human eyeblink elicited by airpuffs, approximately 50 to $100 \mathrm{msec}$, as reported by Kimble (1961). The generally upward intensity-body contraction trend is, of course, in complete accord with similar dynamogenic relationships found for other responses and organisms, e.g., for human electrodermal responses by Kimmel (1967), Sokolov (1963), and Uno and Grings (1965), for human eyeblinks by Passey (1948), and for dogs' salivary reactions by Pavlov (1927).

On the basis of the data shown in Figures 1 and 2, we feel confident in asserting that the present videotape technique for quantifying the elicited reactions of planaria provides a basis for greatly increased sophistication in behavioral research with this organism. Studies of the trial-by-trial course of responding to conditioned and unconditioned stimuli during habituation, conditioning, and experimental extinction, under a wide variety of parametric conditions, now appear entirely feasible and potentially quite fruitful. Further development of the videotape method may be expected also to yield useful information regarding the form of this organism's elicited behavior.

\section{REFERENCES}

Hind, J. E., Goldberg, J. M., Greenwood, D. D., \& Rose, J. E. Some discharge characteristics of single neurons in the inferior colliculus of the cat. II. Timing of the discharges and observations on binaural stimulation. Journal of Neurophysiology, 1963, 26. 321-341.

KIMBLe, G. Hilgard and Marquis' conditioning and learning. New York: Appleton-Century-Crofts, 1961.

KIMmeL, H. D. Equating stimulus intensities by means of the GSR. Psychonomic Science, 1967, 7, 77-78.

Kimmel, H. D., RAY, R. L., \& King, J. A television technique for quantifying conditioned and unconditioned responses of planaria. Behavior Research Methods \& Instrumentation, 1975, 7, 353-356.

PASSEY, G. E. The influence of intensity of unconditioned stimulus upon acquisition of a conditioned response. Journal of Experimental Psychology, 1948, 38, 420-428.

Pavlov, I. P. Conditioned reflexes. London: Oxford University Press, 1927.

Sokolov, Ye. N. Perception and the conditioned reflex. New York: MacMillan, 1963.

Uno, T., \& GRINGs, W. W. Autonomic components of orienting behavior. Psychophysiology, 1965, 1, 311-321.

\section{NOTES}

1. The output gain control on the oscillator was used during data collection to establish what we assumed were equal intensity steps. Subsequent measurement of actual intensities across the electrodes in the water indicated, however, that we did not quite achieve a linear scale.

2. The four animals were run in two completely independent replications, each with two animals, separated by 4 months. The experimental effects reported here did not differ from replication to replication in any noteworthy fashion, and the data of the two replications are therefore combined.

(Received for publication March 8, 1976; revision accepted May 7, 1976.) 